Supplement of Atmos. Chem. Phys., 16, 325-341, 2016

http://www.atmos-chem-phys.net/16/325/2016/

doi:10.5194/acp-16-325-2016-supplement

(C) Author(s) 2016. CC Attribution 3.0 License.

(c) (i)

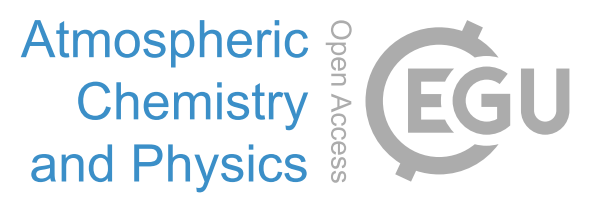

Supplement of

\title{
Nitrogen speciation in various types of aerosols in spring over the northwestern Pacific Ocean
}

\section{Luo et al.}

Correspondence to: S. J. Kao (sjkao@xmu.edu.cn)

The copyright of individual parts of the supplement might differ from the CC-BY 3.0 licence. 
1 Table S1. Sample information, including starting and end time of sampling and

2 location (longitude and latitude).

\begin{tabular}{|c|c|c|c|c|c|c|}
\hline \multirow{2}{*}{ NO. } & \multicolumn{3}{|c|}{ Start } & \multicolumn{3}{|c|}{ End } \\
\hline & Data & Longitude & Latitude & Data & Longitude & Latitude \\
\hline 1 & 0317 13:20 & $120.86 \mathrm{E}$ & $35.85 \mathrm{~N}$ & 0317 19:00 & $121.85 \mathrm{E}$ & $35.51 \mathrm{~N}$ \\
\hline 2 & 0317 19:00 & $121.85 \mathrm{E}$ & $35.51 \mathrm{~N}$ & 0318 07:00 & $123.86 \mathrm{E}$ & $34.28 \mathrm{~N}$ \\
\hline 3 & 0318 07:00 & $123.86 \mathrm{E}$ & $34.28 \mathrm{~N}$ & 0318 19:50 & $125.93 \mathrm{E}$ & $32.96 \mathrm{~N}$ \\
\hline 4 & 0318 19:50 & $125.93 \mathrm{E}$ & $32.96 \mathrm{~N}$ & 0319 07:20 & $127.72 \mathrm{E}$ & $31.82 \mathrm{~N}$ \\
\hline 5 & 0319 07:20 & $127.72 \mathrm{E}$ & $31.82 \mathrm{~N}$ & 0319 18:50 & $129.65 \mathrm{E}$ & $30.97 \mathrm{~N}$ \\
\hline 6 & 0319 19:00 & $129.65 \mathrm{E}$ & $30.97 \mathrm{~N}$ & $032018: 30$ & $134.55 \mathrm{E}$ & $31.00 \mathrm{~N}$ \\
\hline 7 & 0320 18:40 & $134.55 \mathrm{E}$ & $31.00 \mathrm{~N}$ & $032107: 20$ & $136.70 \mathrm{E}$ & $31.05 \mathrm{~N}$ \\
\hline 8 & $032107: 20$ & $136.70 \mathrm{E}$ & $31.05 \mathrm{~N}$ & $032120: 40$ & $137.77 \mathrm{E}$ & $30.63 \mathrm{~N}$ \\
\hline 9 & 0321 20:40 & $137.77 \mathrm{E}$ & $30.63 \mathrm{~N}$ & 0322 07:20 & $139.00 \mathrm{E}$ & $30.48 \mathrm{~N}$ \\
\hline 10 & 0322 11:00 & $139.54 \mathrm{E}$ & $30.48 \mathrm{~N}$ & 0322 20:20 & $141.17 \mathrm{E}$ & $30.04 \mathrm{~N}$ \\
\hline 11 & 0322 20:20 & $141.17 \mathrm{E}$ & $30.04 \mathrm{~N}$ & 0323 07:00 & $142.61 \mathrm{E}$ & $29.48 \mathrm{~N}$ \\
\hline 12 & 0323 07:00 & $142.61 \mathrm{E}$ & $29.48 \mathrm{~N}$ & 0323 19:40 & $145.08 \mathrm{E}$ & $29.65 \mathrm{~N}$ \\
\hline 13 & 0323 19:40 & $145.08 \mathrm{E}$ & $29.65 \mathrm{~N}$ & 0324 07:20 & $146.67 \mathrm{E}$ & $29.59 \mathrm{~N}$ \\
\hline 14 & 0324 07:20 & $146.67 \mathrm{E}$ & $29.59 \mathrm{~N}$ & 0324 19:00 & $148.66 \mathrm{E}$ & $29.17 \mathrm{~N}$ \\
\hline 15 & $032519: 30$ & $148.66 \mathrm{E}$ & $29.17 \mathrm{~N}$ & $032607: 20$ & $148.00 \mathrm{E}$ & $30.22 \mathrm{~N}$ \\
\hline 16 & 0326 09:10 & $148.00 \mathrm{E}$ & $30.22 \mathrm{~N}$ & $032620: 15$ & $148.09 \mathrm{E}$ & $29.58 \mathrm{~N}$ \\
\hline 17 & $032620: 20$ & $148.09 \mathrm{E}$ & $29.58 \mathrm{~N}$ & 0327 08:00 & $147.28 \mathrm{E}$ & $29.25 \mathrm{~N}$ \\
\hline 18 & 0327 08:00 & $147.28 \mathrm{E}$ & $29.25 \mathrm{~N}$ & 0327 17:50 & $146.92 \mathrm{E}$ & $29.00 \mathrm{~N}$ \\
\hline 19 & 0327 19:30 & $146.92 \mathrm{E}$ & $29.00 \mathrm{~N}$ & $03288: 50$ & $148.08 \mathrm{E}$ & $29.00 \mathrm{~N}$ \\
\hline 20 & $04019: 20$ & $148.57 \mathrm{E}$ & $28.85 \mathrm{~N}$ & 0401 18:10 & $149.91 \mathrm{E}$ & $29.75 \mathrm{~N}$ \\
\hline 21 & $040511: 50$ & $148.51 \mathrm{E}$ & $30.22 \mathrm{~N}$ & 0405 19:00 & $148.92 \mathrm{E}$ & $31.31 \mathrm{~N}$ \\
\hline 22 & 0405 19:00 & $148.92 \mathrm{E}$ & $31.31 \mathrm{~N}$ & 0406 09:50 & $147.86 \mathrm{E}$ & $33.01 \mathrm{~N}$ \\
\hline 23 & 0406 09:50 & $147.86 \mathrm{E}$ & $33.01 \mathrm{~N}$ & 0406 18:30 & $147.05 \mathrm{E}$ & $33.00 \mathrm{~N}$ \\
\hline 24 & 0407 09:20 & $145.97 \mathrm{E}$ & $32.99 \mathrm{~N}$ & 0407 22:10 & $145.02 \mathrm{E}$ & $33.95 \mathrm{~N}$ \\
\hline 25 & 0408 17:50 & $145.50 \mathrm{E}$ & $34.00 \mathrm{~N}$ & 0409 08:00 & $147.66 \mathrm{E}$ & $34.00 \mathrm{~N}$ \\
\hline
\end{tabular}




\begin{tabular}{lllllll}
26 & $040908: 00$ & $147.66 \mathrm{E}$ & $34.00 \mathrm{~N}$ & $040919: 10$ & $147.57 \mathrm{E}$ & $33.50 \mathrm{~N}$ \\
27 & $040919: 10$ & $147.57 \mathrm{E}$ & $33.50 \mathrm{~N}$ & $041009: 20$ & $146.51 \mathrm{E}$ & $33.52 \mathrm{~N}$ \\
28 & $041009: 20$ & $146.51 \mathrm{E}$ & $33.52 \mathrm{~N}$ & $041017: 50$ & $145.53 \mathrm{E}$ & $33.50 \mathrm{~N}$ \\
29 & $041019: 30$ & $145.53 \mathrm{E}$ & $33.50 \mathrm{~N}$ & $041105: 40$ & $145.00 \mathrm{E}$ & $33.00 \mathrm{~N}$ \\
30 & $041105: 40$ & $145.00 \mathrm{E}$ & $33.00 \mathrm{~N}$ & $041119: 30$ & $145.01 \mathrm{E}$ & $34.64 \mathrm{~N}$ \\
31 & $041119: 30$ & $145.01 \mathrm{E}$ & $34.64 \mathrm{~N}$ & $041208: 20$ & $145.00 \mathrm{E}$ & $35.46 \mathrm{~N}$ \\
32 & $041208: 20$ & $145.00 \mathrm{E}$ & $35.46 \mathrm{~N}$ & $041219: 00$ & $143.06 \mathrm{E}$ & $36.32 \mathrm{~N}$ \\
33 & $041219: 00$ & $143.06 \mathrm{E}$ & $36.32 \mathrm{~N}$ & $041309: 10$ & $144.60 \mathrm{E}$ & $36.99 \mathrm{~N}$ \\
34 & $041309: 10$ & $144.60 \mathrm{E}$ & $36.99 \mathrm{~N}$ & $041321: 00$ & $144.01 \mathrm{E}$ & $36.00 \mathrm{~N}$ \\
35 & $041409: 10$ & $145.44 \mathrm{E}$ & $34.57 \mathrm{~N}$ & $041417: 20$ & $144.00 \mathrm{E}$ & $34.00 \mathrm{~N}$ \\
36 & $041511: 20$ & $143.97 \mathrm{E}$ & $31.98 \mathrm{~N}$ & $041522: 20$ & $145.49 \mathrm{E}$ & $32.00 \mathrm{~N}$ \\
37 & $041608: 30$ & $145.49 \mathrm{E}$ & $32.00 \mathrm{~N}$ & $041718: 10$ & $141.92 \mathrm{E}$ & $31.00 \mathrm{~N}$ \\
38 & $041718: 10$ & $141.92 \mathrm{E}$ & $31.00 \mathrm{~N}$ & $041809: 10$ & $139.14 \mathrm{E}$ & $31.00 \mathrm{~N}$ \\
39 & $041809: 10$ & $139.14 \mathrm{E}$ & $31.00 \mathrm{~N}$ & $041823: 50$ & $136.43 \mathrm{E}$ & $31.00 \mathrm{~N}$ \\
40 & $041823: 50$ & $136.43 \mathrm{E}$ & $31.00 \mathrm{~N}$ & $041912: 00$ & $134.00 \mathrm{E}$ & $31.00 \mathrm{~N}$ \\
41 & $041912: 00$ & $134.00 \mathrm{E}$ & $31.00 \mathrm{~N}$ & $040923: 50$ & $131.57 \mathrm{E}$ & $30.98 \mathrm{~N}$ \\
42 & $041923: 50$ & $131.57 \mathrm{E}$ & $30.98 \mathrm{~N}$ & $042015: 50$ & $128.65 \mathrm{E}$ & $31.23 \mathrm{~N}$ \\
43 & $042108: 00$ & $126.13 \mathrm{E}$ & $32.44 \mathrm{~N}$ & $042118: 00$ & $124.49 \mathrm{E}$ & $33.87 \mathrm{~N}$ \\
44 & $042118: 10$ & $124.49 \mathrm{E}$ & $33.87 \mathrm{~N}$ & $042209: 20$ & $122.08 \mathrm{E}$ & $35.38 \mathrm{~N}$ \\
\hline & & & & & &
\end{tabular}



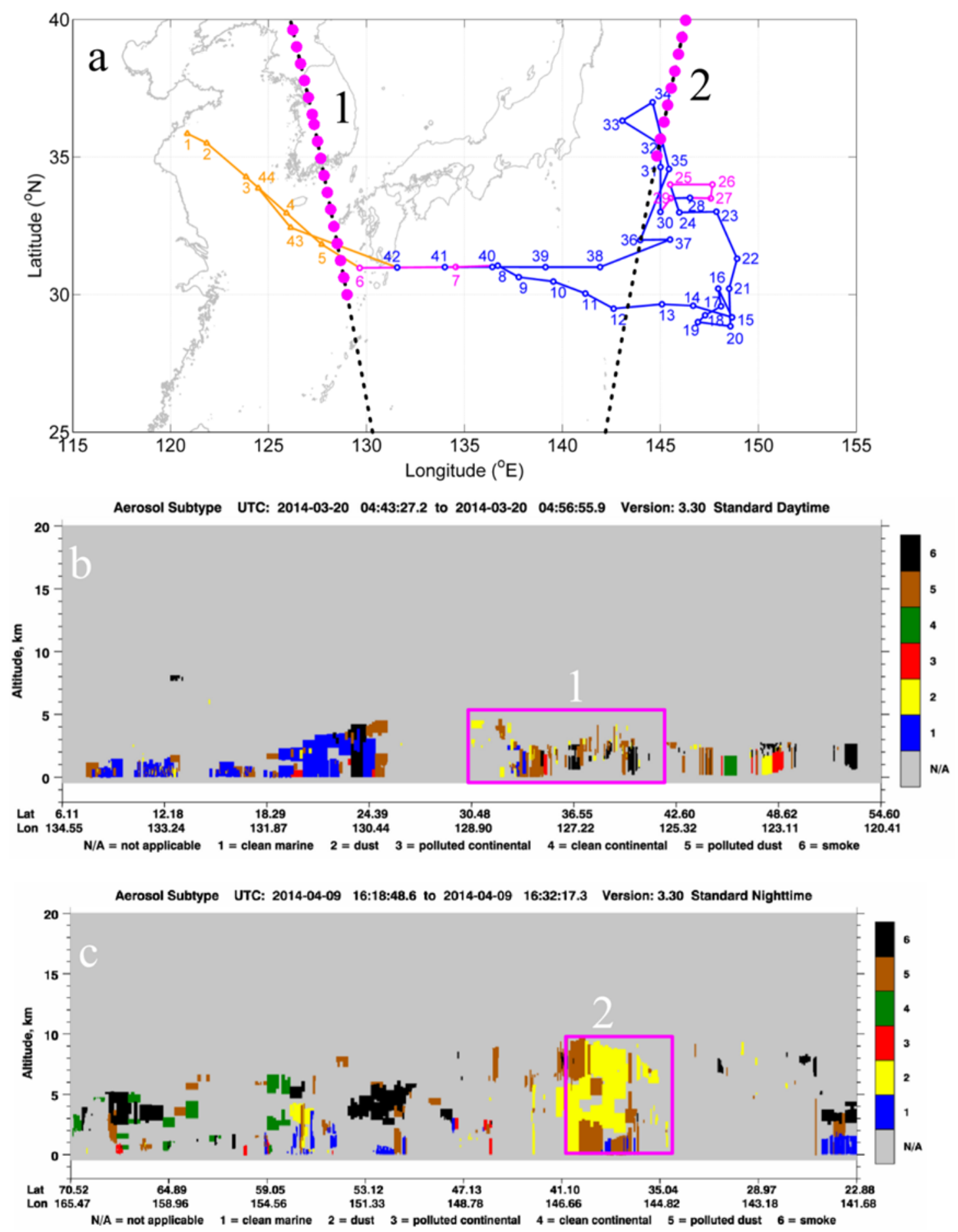

3 Figure S1. Lidar browse images from the NASA cover the NWPO on March 20 and 4 April 9, 2014. Black dashed lines in (a) represent satellite scanning tracks \#1 and \#2.

5 Enlarged pink dots are for the target areas shown in (b) and (c) along the tracks \#1 and

6 \#2, respectively. The pink box in image (b) revealed signals of polluted dust, smoke 7 and dust; while in (c) dust and polluted dust were observed. Both dusty zones locate 8 near the dust aerosol (small open pink dots) we collected along the cruise track. 